PROCEEDINGS OF THE

AMERICAN MATHEMATICAL SOCIETY

Volume 132, Number 7, Pages 2085-2094

S 0002-9939(04)07305-8

Article electronically published on February 6, 2004

\title{
FINITE AOMOTO-ITO-MACDONALD SUMS
}

\author{
ANA-CECILIA DE LA MAZA
}

(Communicated by John R. Stembridge)

\begin{abstract}
We present finite truncations of the Aomoto-Ito-Macdonald sums associated with root systems through a two-step reduction procedure. The first reduction restricts the sum from the root lattice to a Weyl chamber; the second reduction arises after imposing a truncation condition on the parameters, and gives rise to a finite sum over a Weyl alcove.
\end{abstract}

\section{INTRODUCTION}

The study of summation formulas for hypergeometric and basic hypergeometric series has a long and rich history GR. The general structure of most summation formulas is that of a closed expression for a (basic) hypergeometric series - at a special value of the argument-in terms of products of $(q$-)gamma functions. In recent work, Aomoto and Ito considered analogous product formulas for certain multivariate basic hypergeometric series associated with the crystallographic root systems [A], K], I1], I2], [I3. These sums may be interpreted as evaluation formulas for Selberg-type $q$-Jackson integrals associated with root systems [AAR, [M3], [], A], I2, or alternatively, as affine analogues of well-known product formulas due to Macdonald for the Poincaré series of Weyl groups M1, M2, M4. We will refer to the multivariate basic hypergeometric summation formulas in question as Aomoto-Ito-Macdonald sums. Various other types of multivariate (basic) hypergeometric summation formulas can be found in the works of Milne, Gustafson, Schlosser, and Rosengren [M], G1, [G2], [ML, [S], R].

The purpose of this note is to exhibit a finite analogue of the Aomoto-ItoMacdonald sum. To this end we perform a two-step reduction procedure. The first reduction restricts the sum from the root lattice to a Weyl chamber; the second reduction arises after imposing a truncation condition on the parameters, and gives rise to a finite sum over a Weyl alcove. For the type $A$ root systems, the corresponding reductions of the Aomoto-Ito-Macdonald sums were already studied in [DV] in connection with the eigenvalue problem of the compactified trigonometric quantum Ruijsenaars-Schneider model. More specifically, the results reported

Received by the editors December 13, 2002 and, in revised form, April 3, 2003 and April 11, 2003.

2000 Mathematics Subject Classification. Primary 33D67; Secondary 05A19, 11L03.

Key words and phrases. Multivariate basic hypergeometric series, summation formulas, root systems.

This work was supported in part by the Fondo Nacional de Desarrollo Científico y Tecnológico (FONDECYT) Grant \# 1010205 and the Programa formas cuadráticas of the Universidad de Talca.

(C)2004 American Mathematical Society 
below constitute a generalization of the finite Aomoto-Ito-Macdonald sum of [DV] to the case of an arbitrary reduced root system. For analogous summation formulas connected with the nonreduced root systems the reader is referred to the papers [D], [DS].

Note. We will employ the following standard notational conventions from the theory of basic hypergeometric series $[\mathrm{GR}$. For $m \in \mathbb{Z}$ and $a, q \in \mathbb{C}$, the $q$-shifted factorial is defined as

$$
(a ; q)_{m}=\left\{\begin{array}{lll}
(1-a)(1-a q) \cdots\left(1-a q^{m-1}\right) & \text { if } & m=1,2,3, \ldots, \\
1 & \text { if } & m=0 \\
\frac{1}{\left(1-a q^{-1}\right)\left(1-a q^{2}\right) \ldots\left(1-a q^{-m}\right)} & \text { if } & m=-1,-2,-3, \ldots
\end{array}\right.
$$

where it is assumed that $a q^{-k} \neq 1$ for $1 \leq k \leq-m$ if $m$ is negative. For $|q|<1$, the definition of the $q$-shifted factorial may be extended to include the case $m=\infty$ through limit transition $(a ; q)_{\infty}:=\lim _{m \rightarrow \infty}(a ; q)_{m}=\prod_{k=0}^{\infty}\left(1-a q^{k}\right)$. Products of $q$-shifted factorials will often be abbreviated as

$$
\left(a_{1}, a_{2}, \ldots, a_{k} ; q\right)_{m}:=\left(a_{1} ; q\right)_{m}\left(a_{2} ; q\right)_{m} \cdots\left(a_{k} ; q\right)_{m} .
$$

\section{The Aомoto-Ito-Macdonald sum}

Let $\mathbf{E},\langle\cdot, \cdot\rangle$ be a real finite-dimensional Euclidean vector space spanned by an irreducible crystallographic root system $R$ (see, e.g., [B] for preliminaries on root systems). Throughout this note, it will always be assumed that $R$ be reduced. We will denote the root lattice by $\mathcal{Q}:=\operatorname{Span}_{\mathbb{Z}}(R)$ and the weight lattice by $\mathcal{P}:=\{\lambda \in$ $\left.\mathbf{E} \mid\left\langle\alpha^{\vee}, \lambda\right\rangle \in \mathbb{Z}, \forall \alpha \in R\right\}$, where $\alpha^{\vee}:=2 \alpha /\langle\alpha, \alpha\rangle$. To a choice of positive roots $R^{+}$, we associate a weighted half-sum of the positive (co)roots as

$$
\rho_{g}:=\frac{1}{2} \sum_{\alpha \in R^{+}} g_{\alpha} \alpha, \quad \rho_{g}^{\vee}:=\frac{1}{2} \sum_{\alpha \in R^{+}} g_{\alpha} \alpha^{\vee},
$$

where $g_{\alpha} \in \mathbb{C}$ depends only on the length of the root $\alpha$. More specifically, one has that $g_{\alpha}=g, \forall \alpha \in R$ if all roots have the same length (i.e., when the root system is simply-laced), and otherwise one has that $g_{\alpha}$ is equal to $g_{l}$ or $g_{s}$, respectively, depending on whether $\alpha$ is a long or a short root. Let $S \subset R^{+}$denote the basis of simple roots corresponding to $R^{+}$.

We are now in the position to formulate the Aomoto-Ito-Macdonald sum.

Theorem 1 (Aomoto-Ito-Macdonald Sum [A], [12, [M4]). Let $0<q<1$ and $\operatorname{Re}\left(g_{\alpha}\right)<0$, and let $\mathbf{z} \in \mathbf{E}$ be generic in the sense that $g_{\alpha}+\left\langle\alpha^{\vee}, \mathbf{z}\right\rangle \notin\left(\mathbb{Z}+\frac{2 \pi}{i \log q} \mathbb{Z}\right)$ for all $\alpha \in R^{+}$. Then

$$
\sum_{\mu \in \mathcal{Q}} q^{-2\left\langle\rho_{g}^{\vee}, \mathbf{z}+\mu\right\rangle} \prod_{\alpha \in R^{+}}\left(1-q^{\left\langle\alpha^{\vee}, \mathbf{z}+\mu\right\rangle}\right) \frac{\left(q^{1-g_{\alpha}+\left\langle\alpha^{\vee}, \mathbf{z}+\mu\right\rangle} ; q\right)_{\infty}}{\left(q^{g_{\alpha}+\left\langle\alpha^{\vee}, \mathbf{z}+\mu\right\rangle} ; q\right)_{\infty}}=\theta_{0} \theta(\mathbf{z}),
$$

where

$$
\begin{aligned}
\theta_{0} & =\prod_{\alpha \in R^{+}} \frac{\left(q^{1-g_{\alpha}-\left\langle\alpha, \rho_{g}^{\vee}\right\rangle}, q^{\delta_{\alpha}+g_{\alpha}-\left\langle\alpha, \rho_{g}^{\vee}\right\rangle} ; q\right)_{\infty}}{\left(q^{1-\left\langle\alpha, \rho_{g}^{\vee}\right\rangle}, q^{-\left\langle\alpha, \rho_{g}^{\vee}\right\rangle} ; q\right)_{\infty}} \\
\theta(\mathbf{z}) & =q^{-2\left\langle\rho_{g}^{\vee}, \mathbf{z}\right\rangle} \prod_{\alpha \in R^{+}} \frac{\left(q^{\left\langle\alpha^{\vee}, \mathbf{z}\right\rangle}, q^{1-\left\langle\alpha^{\vee}, \mathbf{z}\right\rangle} ; q\right)_{\infty}}{\left(q^{g_{\alpha}+\left\langle\alpha^{\vee}, \mathbf{z}\right\rangle}, q^{1-g_{\alpha}-\left\langle\alpha^{\vee}, \mathbf{z}\right\rangle} ; q\right)_{\infty}}
\end{aligned}
$$


with

$$
\delta_{\alpha}:= \begin{cases}1 & \text { for } \alpha \in S, \\ 0 & \text { for } \alpha \in R^{+} \backslash S .\end{cases}
$$

In A Aomoto showed that the sum on the 1.h.s. of 2.2a is proportional to $\theta(\mathbf{z})$ (2.2c). The value of $\theta_{0}$ (2.2b was subsequently conjectured by Ito [I2], who verified his conjecture for all root systems of $\operatorname{rank}(:=\operatorname{dim}(\mathbf{E}))$ up to 2 and for the $A_{N}$ series. Soon thereafter, Macdonald found a complete proof of Theorem 1, [M4]. The restrictions on the parameters ensure that all denominators are nonzero and that the series on the l.h.s. converges in absolute value.

For our purposes, it will be more convenient to work with slightly different normalizations of the summation formula. Division by $\theta(\mathbf{z})$ eliminates the $\mathbf{z}$ dependence from the r.h.s., thus leading to the identity

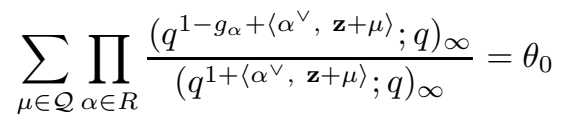

(with $\mathbf{z} \in \mathbf{E}$ such that $\left\langle\alpha^{\vee}, \mathbf{z}\right\rangle \notin \mathbb{Z}+\frac{2 \pi}{i \log q} \mathbb{Z}$ for all $\alpha \in R^{+}$). Furthermore, normalization such that the term corresponding to $\mu=\mathbf{0}$ equals 1 casts the summation formula in the following basic hypergeometric form:

$$
\begin{array}{r}
\sum_{\mu \in \mathcal{Q}} q^{-2\left\langle\rho_{g}^{\vee}, \mu\right\rangle} \prod_{\alpha \in R^{+}}\left(\frac{1-q^{\left\langle\alpha^{\vee}, \mathbf{z}+\mu\right\rangle}}{1-q^{\left\langle\alpha^{\vee}, \mathbf{z}\right\rangle}}\right) \frac{\left(q^{g_{\alpha}+\left\langle\alpha^{\vee}, \mathbf{z}\right\rangle} ; q\right)_{\left\langle\alpha^{\vee}, \mu\right\rangle}}{\left(q^{1-g_{\alpha}+\left\langle\alpha^{\vee}, \mathbf{z}\right\rangle} ; q\right)_{\left\langle\alpha^{\vee}, \mu\right\rangle}} \\
=\theta_{0} \prod_{\alpha \in R} \frac{\left(q^{1+\left\langle\alpha^{\vee}, \mathbf{z}\right\rangle} ; q\right)_{\infty}}{\left(q^{1-g_{\alpha}+\left\langle\alpha^{\vee}, \mathbf{z}\right\rangle} ; q\right)_{\infty}}
\end{array}
$$

(with $\mathbf{z} \in \mathbf{E}$ such that $\left\langle\alpha^{\vee}, \mathbf{z}\right\rangle \notin \frac{2 \pi}{i \log q} \mathbb{Z}$ and $g_{\alpha}-1+\left\langle\alpha^{\vee}, \mathbf{z}\right\rangle \notin \mathbb{N}+\frac{2 \pi}{i \log q} \mathbb{Z}$ for all $\alpha \in R)$.

Remark 2.1. It is clear from the form in 2.3a that we may replace the summation over the root lattice $\mathcal{Q}$ in the Aomoto-Ito-Macdonald sum by a summation over the translated lattice $\sigma+\mathcal{Q}$ with $\sigma$ in the weight lattice $\mathcal{P}$ (by the translational symmetry in $\mathbf{z}$ ). In particular, summation over all translates along $\sigma \in \mathcal{P} / \mathcal{Q} \cong$ $\left\{\mu \in \mathcal{P} \mid 0 \leq\left\langle\alpha^{\vee}, \mu\right\rangle \leq 1, \forall \alpha \in R^{+}\right\}$, then reveals that we may replace the summation over the root lattice $\mathcal{Q}$ by a summation over the weight lattice $\mathcal{P}$ upon multiplication of the r.h.s. by the index $|\mathcal{P} / \mathcal{Q}|$.

Remark 2.2. For a root system of rank 1 , one has that $\mathcal{P} \cong \mathbb{Z}, \mathcal{Q} \cong 2 \mathbb{Z}$, and that $\rho_{g}^{\vee}=g / 2$. The sum in $2.3 \mathrm{~b}$ reduces in this case to the identity

$$
\begin{aligned}
& \sum_{\substack{m \in \mathbb{Z} \\
m \text { even }}} q^{-g m}\left(\frac{1-q^{z+m}}{1-q^{z}}\right) \frac{\left(q^{g+z} ; q\right)_{m}}{\left(q^{1-g+z} ; q\right)_{m}} \\
& \quad=\frac{\left(q, q^{1-2 g} ; q\right)_{\infty}}{\left(q^{1-g}, q^{-g} ; q\right)_{\infty}} \frac{\left(q^{1+z}, q^{1-z} ; q\right)_{\infty}}{\left(q^{1-g+z}, q^{1-g-z} ; q\right)_{\infty}} .
\end{aligned}
$$

In view of the previous remark, this formula remains valid when replacing the summation over the even integers by a summation over the odd integers (and thus also by a summation over all integers upon multiplying the r.h.s. by 2 ). 


\section{Reduction to a Weyl chamber}

Let $\mathcal{P}^{+}$be the dominant cone of weights in (the closure of) the positive Weyl chamber, i.e., $\mathcal{P}^{+}=\left\{\mu \in \mathcal{P} \mid\left\langle\alpha^{\vee}, \mu\right\rangle \geq 0, \forall \alpha \in R^{+}\right\}$. Specialization of $\mathbf{z}$ to the special point $\rho_{g}$ (2.1) reduces the Aomoto-Ito-Macdonald summation formula to a sum over weights $\mu$ inside the dominant cone $\mathcal{P}^{+}$.

Definition. A weight $\sigma \in \mathcal{P}$ is called minuscule if $0 \leq\left\langle\alpha^{\vee}, \sigma\right\rangle \leq 1$ for all $\alpha \in R^{+}$.

Every root system $R$ has a trivial minuscule weight $\sigma=\mathbf{0}$. It is well known that the total number of minuscule weights is equal to the index $|\mathcal{P} / \mathcal{Q}|$ (cf. also Remark 2.11).

Theorem 2 (Reduced Aomoto-Ito-Macdonald Sum). Let $0<q<1$, and let $\operatorname{Re}\left(g_{\alpha}\right)<0$ be such that $g_{\alpha}-\left\langle\alpha^{\vee}, \rho_{g}\right\rangle-1 \notin \mathbb{N}+\frac{2 \pi}{i \log q} \mathbb{Z}$ for all $\alpha \in R^{+}$. Then for any minuscule weight $\sigma$,

$$
\sum_{\mu \in(\sigma+\mathcal{Q}) \cap \mathcal{P}^{+}} q^{-2\left\langle\rho_{g}^{\vee}, \mu\right\rangle} \prod_{\alpha \in R^{+}}\left(\frac{1-q^{\left\langle\alpha^{\vee}, \rho_{g}+\mu\right\rangle}}{1-q^{\left\langle\alpha^{\vee}, \rho_{g}\right\rangle}}\right) \frac{\left(q^{g_{\alpha}+\left\langle\alpha^{\vee}, \rho_{g}\right\rangle} ; q\right)_{\left\langle\alpha^{\vee}, \mu\right\rangle}}{\left(q^{1-g_{\alpha}+\left\langle\alpha^{\vee}, \rho_{g}\right\rangle} ; q\right)_{\left\langle\alpha^{\vee}, \mu\right\rangle}}=\mathcal{N}_{\infty},
$$

with

$$
\mathcal{N}_{\infty}=\prod_{\alpha \in R^{+}} \frac{\left(q^{1+\left\langle\alpha^{\vee}, \rho_{g}\right\rangle} ; q\right)_{\infty}}{\left(q^{-\left\langle\alpha, \rho_{g}^{\vee}\right\rangle} ; q\right)_{\infty}} \prod_{\alpha \in R^{+} \backslash S} \frac{\left(q^{g_{\alpha}-\left\langle\alpha, \rho_{g}^{\vee}\right\rangle} ; q\right)_{\infty}}{\left(q^{1-g_{\alpha}+\left\langle\alpha^{\vee}, \rho_{g}\right\rangle} ; q\right)_{\infty}}
$$

Proof. Our starting point is the Aomoto-Ito-Macdonald sum in the basic hypergeometric normalization of (2.3b) with a translated lattice $\sigma+\mathcal{Q}$ (cf. Remark 2.1). Let us temporarily assume that the parameters $g_{\alpha}$ are such that $\operatorname{Re}\left(g_{\alpha}\right)<0$ and $g_{\alpha}-1+\left\langle\alpha^{\vee}, \rho_{g}\right\rangle \notin \mathbb{N}+\frac{2 \pi}{i \log q} \mathbb{Z}$ for all $\alpha \in R$. Substitution of $\mathbf{z}=\rho_{g}$ then reduces the sum on the l.h.s. to a sum over those weights $\mu$ in the lattice $\sigma+\mathcal{Q}$ that lie inside the dominant cone $\mathcal{P}^{+}$. Indeed, for $\mu \in(\sigma+\mathcal{Q}) \backslash \mathcal{P}^{+}$there exists a simple $\operatorname{root} \beta \in S$, such that $\left\langle\beta^{\vee}, \mu\right\rangle<0$. Hence, we pick up a zero in the corresponding term of the Aomoto-Ito-Macdonald sum (2.3b) from the factor

$$
\frac{1}{\left(q^{1-g_{\beta}+\left\langle\beta^{\vee}, \rho_{g}\right\rangle} ; q\right)_{\left\langle\beta^{\vee}, \mu\right\rangle}}=\frac{1}{(q ; q)_{\left\langle\beta^{\vee}, \mu\right\rangle}}=0 .
$$

The simplification of the corresponding evaluation constant from the r.h.s. of 2.3b to the stated value $\mathcal{N}_{\infty}$ (3.1b) (for $\mathbf{z}=\rho_{g}$ ) hinges on the identity (cf. Remark 3.3 below)

$$
\prod_{\alpha \in R^{+}} \frac{\left(q^{1-\left\langle\alpha^{\vee}, \rho_{g}\right\rangle} ; q\right)_{\infty}}{\left(q^{1-g_{\alpha}-\left\langle\alpha^{\vee}, \rho_{g}\right\rangle} ; q\right)_{\infty}}=\prod_{\alpha \in R^{+}} \frac{\left(q^{1-\left\langle\alpha, \rho_{g}^{\vee}\right\rangle} ; q\right)_{\infty}}{\left(q^{1-g_{\alpha}-\left\langle\alpha, \rho_{g}^{\vee}\right\rangle} ; q\right)_{\infty}}
$$

(and the fact that $\left\langle\alpha^{\vee}, \rho_{g}\right\rangle=\left\langle\alpha, \rho_{g}^{\vee}\right\rangle=g_{\alpha}$ for any $\alpha$ in the simple base $S$ ). In the resulting summation formula we may relax the restrictions on the parameters $g_{\alpha}$ somewhat through analytic continuation; this recovers the parameter conditions stated in the theorem.

The product formula for the evaluation constant $\mathcal{N}_{\infty}$ in Theorem[2]contains some redundancy. In Table 1 we have listed - for each reduced irreducible root system $R$ - a more compact expression for $\mathcal{N}_{\infty}$. These formulas are obtained from (3.1b) through cancellation of the common factors in the numerator and denominator. For the reader's convenience, we have also included the index $|\mathcal{P} / \mathcal{Q}|$ in each case. 


\begin{tabular}{|c|c|c|}
\hline$R$ & $\mathcal{N}_{\infty}$ & $|\mathcal{P} / \mathcal{Q}|$ \\
\hline$A_{N}$ & $\prod_{k=1}^{N} \frac{\left(q^{1+k g} ; q\right)_{\infty}}{\left(q^{-k g} ; q\right)_{\infty}}$ & $N+1$ \\
\hline$B_{N}$ & $\prod_{k=1}^{N} \frac{\left(q^{1+(N+k-2) g_{l}+g_{s}} ; q\right)_{\infty}}{\left(q^{-(N-k) g_{l}-g_{s}} ; q\right)_{\infty}} \prod_{k=1}^{\left[\frac{N}{2}\right]} \frac{\left(q^{1+(2 k-1) g_{l}} ; q\right)_{\infty}}{\left(q^{-(2 N-2 k-1) g_{l}-2 g_{s}} ; q\right)_{\infty}} \prod_{k=1}^{\left[\frac{N}{2}\right]} \frac{\left(q^{-2(k-1) g_{l}-2 g_{s}} ; q\right)_{\infty}}{\left(q^{1+2(N-k) g_{l}} ; q\right)_{\infty}}$ & 2 \\
\hline$C_{N}$ & $\prod_{k=1}^{N} \frac{\left(q^{1+(k-1) g_{s}+g_{l}} ; q\right)_{\infty}}{\left(q^{-(2 N-k-1) g_{s}-g_{l}} ; q\right)_{\infty}} \prod_{k=1}^{\left[\frac{N}{2}\right]} \frac{\left(q^{1+(2 N-2 k-1) g_{s}+2 g_{l}} ; q\right)_{\infty}}{\left(q^{-(2 k-1) g_{s}} ; q\right)_{\infty}} \prod_{k=1}^{\left[\frac{N}{2}\right]} \frac{\left(q^{-2(N-k) g_{s}} ; q\right)_{\infty}}{\left(q^{1+2(k-1) g_{s}+2 g l} ; q\right)_{\infty}}$ & 2 \\
\hline$D_{N}$ & $\frac{\left(q^{1+(N-1) g} ; q\right)_{\infty}}{\left(q^{-(N-1) g} ; q\right)_{\infty}} \prod_{k=1}^{N-1} \frac{\left(q^{1+(2 k-1) g} ; q\right)_{\infty}}{\left(q^{-(2 k-1) g} ; q\right)_{\infty}}$ & 4 \\
\hline$E_{6}$ & $\frac{\left(q^{1+g}, q^{1+4 g}, q^{1+5 g}, q^{1+7 g}, q^{1+8 g}, q^{1+11 g} ; q\right)_{\infty}}{\left(q^{-g}, q^{-4 g}, q^{-5 g}, q^{-7 g}, q^{-8 g}, q^{-11 g} ; q\right)_{\infty}}$ & 3 \\
\hline$E_{7}$ & $\frac{\left(q^{1+g}, q^{1+5 g}, q^{1+7 g}, q^{1+9 g}, q^{1+11 g}, q^{1+13 g}, q^{1+17 g} ; q\right)_{\infty}}{\left(q^{-g}, q^{-5 g}, q^{-7 g}, q^{-9 g}, q^{-11 g}, q^{-13 g}, q^{-17 g} ; q\right)_{\infty}}$ & 2 \\
\hline$E_{8}$ & $\frac{\left(q^{1+g}, q^{1+7 g}, q^{1+11 g}, q^{1+13 g}, q^{1+17 g}, q^{1+19 g}, q^{1+23 g}, q^{1+29 g} ; q\right)_{\infty}}{\left(q^{-g}, q^{-7 g}, q^{-11 g}, q^{-13 g}, q^{-17 g}, q^{-19 g}, q^{-23 g}, q^{-29 g} ; q\right)_{\infty}}$ & 1 \\
\hline$F_{4}$ & $\frac{\left(q^{-4 g_{s}}, q^{-6 g_{s}-2 g_{l}}, q^{1+g_{l}}, q^{1+g_{s}+3 g_{l}}, q^{1+2 g_{s}+3 g_{l}}, q^{1+3 g_{s}+4 g_{l}}, q^{1+3 g_{s}+5 g_{l}}, q^{1+5 g_{s}+6 g_{l}} ; q\right)_{\infty}}{\left(q^{1+2 g_{s}+6 g_{l}}, q^{1+4 g_{l}}, q^{-6 g_{s}-5 g_{l}}, q^{-5 g_{s}-3 g_{l}}, q^{-4 g_{s}-3 g_{l}}, q^{-3 g_{s}-2 g_{l}}, q^{-3 g_{s}-g_{l}}, q^{-g_{s}} ; q\right)_{\infty}}$ & 1 \\
\hline$G_{2}$ & $\frac{\left(q^{1+g_{l}}, q^{1+g_{s}+2 g_{l}}, q^{1+2 g_{s}+3 g_{l}}, q^{-3 g_{s}} ; q\right)_{\infty}}{\left(q^{1+3 g_{l}}, q^{-g_{s}}, q^{-2 g_{s}-g_{l}}, q^{-3 g_{s}-2 g_{l}} ; q\right)_{\infty}}$ & 1 \\
\hline
\end{tabular}

TABLE 1. Reduced Expressions for $\mathcal{N}_{\infty}$

Remark 3.1. For the simply-laced root systems (i.e., the types $A, D, E$ ) and for the nonsimply-laced root systems (i.e., the types $B, C, F, G$ ) with $g_{l}=g_{s}$, we have that $g_{\alpha}=g, \forall \alpha \in R$. The formula in Table 1 is in these cases of the form

$$
\mathcal{N}_{\infty}=\prod_{k=1}^{N} \frac{\left(q^{1+g m_{k}} ; q\right)_{\infty}}{\left(q^{-g m_{k}} ; q\right)_{\infty}}
$$

where $N:=\operatorname{dim}(\mathbf{E})$ denotes the rank and $m_{1}, \ldots, m_{N}$ are the exponents of (the Weyl group of) the root system $R$.

Remark 3.2. For a root system of rank $N=1$, one has that $\rho_{g}=g, \mathcal{P}^{+} \cong \mathbb{N}$ and $\sigma \in\{0,1\}$ (cf. Remark [2.2). The sum in Theorem 2 reduces in this case to the 
identity

$$
\sum_{\substack{m \in \mathbb{N} \\ m \text { even/odd }}} q^{-g m}\left(\frac{1-q^{g+m}}{1-q^{g}}\right) \frac{\left(q^{2 g} ; q\right)_{m}}{(q ; q)_{m}}=\frac{\left(q^{1+g} ; q\right)_{\infty}}{\left(q^{-g} ; q\right)_{\infty}}
$$

where the choice of summation over the even or odd nonnegative integers corresponds to the choice of $\sigma$ equal to 0 or 1 , respectively.

Remark 3.3. The identity (3.2) in the proof of Theorem 2 is trivial when the root system is simply-laced (whence $R^{\vee}=R$ ). For the nonsimply-laced root systems, we checked the identity in question on a case-by-case basis through direct computation.

\section{Reduction to a Weyl alcove}

For parameters subject to a truncation condition, the reduced Aomoto-ItoMacdonald sum of Theorem 2 truncates to a finite sum over an alcove inside the dominant cone $\mathcal{P}^{+}$. Let $\alpha_{0}$ be the (positive) root in $R$ such that $\alpha_{0}^{\vee}$ is the maximal coroot of $R^{\vee}:=\left\{\alpha^{\vee} \mid \alpha \in R\right\}$. We define

$$
g_{R}:=g_{\alpha_{0}}+\left\langle\alpha_{0}^{\vee}, \rho_{g}\right\rangle
$$

and, for $M \in \mathbb{N}$,

$$
\mathcal{P}_{M}^{+}:=\left\{\mu \in \mathcal{P}^{+} \mid\left\langle\alpha_{0}^{\vee}, \mu\right\rangle \leq M\right\} .
$$

Theorem 3 (Terminating Aomoto-Ito-Macdonald Sum). Let $M \in \mathbb{N} \backslash\{0\}$ and $g_{\alpha}>0, \forall \alpha \in R$. Then for

$$
q=\exp \left(\frac{2 \pi i}{g_{R}+M}\right)
$$

and any minuscule weight $\sigma$,

$$
\sum_{\mu \in(\sigma+\mathcal{Q}) \cap \mathcal{P}_{M}^{+}} q^{-\left\langle\rho_{g}^{\vee}, \mu\right\rangle} \prod_{\alpha \in R^{+}}\left(\frac{1-q^{\left\langle\alpha^{\vee}, \rho_{g}+\mu\right\rangle}}{1-q^{\left\langle\alpha^{\vee}, \rho_{g}\right\rangle}}\right) \frac{\left(q^{g_{\alpha}+\left\langle\alpha^{\vee}, \rho_{g}\right\rangle} ; q\right)_{\left\langle\alpha^{\vee}, \mu\right\rangle}}{\left(q^{1-g_{\alpha}+\left\langle\alpha^{\vee}, \rho_{g}\right\rangle} ; q\right)_{\left\langle\alpha^{\vee}, \mu\right\rangle}}=\mathcal{N}_{M},
$$

where

$$
\mathcal{N}_{M}=\frac{\prod_{\alpha \in R^{+}}\left(q^{1+\left\langle\alpha^{\vee}, \rho_{g}\right\rangle} ; q\right)_{M-1}}{\prod_{\alpha \in R^{+} \backslash S}\left(q^{1-g_{\alpha}+\left\langle\alpha^{\vee}, \rho_{g}\right\rangle} ; q\right)_{M-1}} .
$$

Proof. Let us first pick parameters from the domain described in Theorem2 subject to the additional constraint that

$$
g_{R}+M=2 \pi i / \log (q) .
$$

The reduced Aomoto-Ito-Macdonald sum then truncates to a finite sum over the alcove $\mathcal{P}_{M}^{+}$. Indeed, for $\mu \in \mathcal{P}^{+} \backslash \mathcal{P}_{M}^{+}$we have that $\left\langle\alpha_{0}^{\vee}, \mu\right\rangle>M$, whence we pick up a zero in the corresponding term on the l.h.s. from the factor

$$
\left(q^{g_{\alpha_{0}}+\left\langle\alpha_{0}^{\vee}, \rho_{g}\right\rangle} ; q\right)_{\left\langle\alpha_{0}^{\vee}, \mu\right\rangle}=\left(q^{g_{R}} ; q\right)_{\left\langle\alpha_{0}^{\vee}, \mu\right\rangle}=\left(q^{-M} ; q\right)_{\left\langle\alpha_{0}^{\vee}, \mu\right\rangle}=0 .
$$


The reduction of the evaluation constant $\mathcal{N}_{\infty}$ on the r.h.s. follows from the identities

$$
\begin{aligned}
& \prod_{\alpha \in R^{+}} \frac{\left(q^{1+\left\langle\alpha^{\vee}, \rho_{g}\right\rangle} ; q\right)_{\infty}}{\left(q^{-\left\langle\alpha, \rho_{g}^{\vee}\right\rangle} ; q\right)_{\infty}} \prod_{\alpha \in R^{+} \backslash S} \frac{\left(q^{g_{\alpha}-\left\langle\alpha, \rho_{g}^{\vee}\right\rangle} ; q\right)_{\infty}}{\left(q^{1-g_{\alpha}+\left\langle\alpha^{\vee}, \rho_{g}\right\rangle} ; q\right)_{\infty}} \\
= & \prod_{\alpha \in R^{+}} \frac{\left(q^{1+\left\langle\alpha^{\vee}\right.}, \rho_{g}\right\rangle}{\left(q^{M+g_{R}-\left\langle\alpha, \rho_{g}^{\vee}\right\rangle} ; q\right)_{\infty}} \prod_{\alpha \in R^{+} \backslash S} \frac{\left(q^{M+g_{R}+g_{\alpha}-\left\langle\alpha, \rho_{g}^{\vee}\right\rangle} ; q\right)_{\infty}}{\left(q^{1-g_{\alpha}+\left\langle\alpha^{\vee}, \rho_{g}\right\rangle} ; q\right)_{\infty}} \\
= & \prod_{\alpha \in R^{+}} \frac{\left(q^{1+\left\langle\alpha^{\vee}, \rho_{g}\right\rangle} ; q\right)_{\infty}}{\left(q^{M+\left\langle\alpha^{\vee}, \rho_{g}\right\rangle} ; q\right)_{\infty}} \prod_{\alpha \in R^{+} \backslash S} \frac{\left(q^{M-g_{\alpha}+\left\langle\alpha^{\vee}, \rho_{g}\right\rangle} ; q\right)_{\infty}}{\left(q^{1-g_{\alpha}+\left\langle\alpha^{\vee}, \rho_{g}\right\rangle} ; q\right)_{\infty}},
\end{aligned}
$$

where the second equality follows from a straightforward but tedious case-by-case computation for each root system. The resulting formula on the third line simplifies to the stated expression for $\mathcal{N}_{M}$ upon cancelling the common tails of the infinite products. This proves the terminating Aomoto-Ito-Macdonald sum for $q=$ $\exp \left(2 \pi i /\left(g_{R}+M\right)\right)$ with parameters $g_{\alpha}$ such that $\operatorname{Re}\left(g_{\alpha}\right)<0$ and $\operatorname{Re}\left(g_{R}\right)=-M$, $\operatorname{Im}\left(g_{R}\right)<0$. By analytic continuation, we may now extend the identity to a parameter domain of generic complex values for $g_{\alpha}$. The restriction to the parameter domain of positive real values for $g_{\alpha}$ stated in the theorem ensures that all numerators and denominators are nonzero.

Elimination of the redundant factors in $\mathcal{N}_{M}$ (4.3c) now gives rise to the reduced expressions in Table 2. For the reader's convenience, we have also listed the corresponding values of $g_{R}$ (4.1) in each case.

Remark 4.1. In Theorem3 we have (see (4.3a)) that $q=e^{i \theta}$ with $\theta=2 \pi /\left(g_{R}+M\right) \in$ $(0,2 \pi)$. The powers of the form $q^{w}, w \in \mathbb{R}$ appearing in Eqs. (4.3b), (4.3c) are to be chosen in accordance with the principal branch in the sense that $q^{w}:=e^{i \theta w}$.

Remark 4.2. The reduction from $\mathcal{N}_{\infty}$ to $\mathcal{N}_{M}$ for $q=\exp \left(2 \pi i /\left(g_{R}+M\right)\right)$ is in fact more easily performed on a case-by-case basis at the level of the reduced expressions in Table 1 and Table 2 (rather than directly with the aid of the uniform expressions in Theorem 2 and Theorem 3 ).

Remark 4.3. The restriction to positive values for $g_{\alpha}$ in Theorem 3 ensures that the terms of the basic hypergeometric series on the l.h.s. are all positive (cf. Remark 4.7 below). By analytic continuation, the summation identity can be extended to a domain of generic complex values for the parameters $g_{\alpha}$ (cf. the proof of Theorem 3).

Remark 4.4. If $g_{\alpha}=g, \forall \alpha \in R$, then the product formula for $\mathcal{N}_{M}$ in Table 2 amounts to the following expression in terms of the exponents $m_{k}$ (see also Remark 3.1)

$$
\mathcal{N}_{M}=\prod_{k=1}^{N}\left(q^{1+g m_{k}} ; q\right)_{M-1}
$$




\begin{tabular}{|c|c|c|}
\hline$R$ & $\mathcal{N}_{M}$ & $g_{R}$ \\
\hline$A_{N}$ & $\prod_{k=1}^{N}\left(q^{1+k g} ; q\right)_{M-1}$ & $(N+1) g$ \\
\hline$B_{N}$ & $\frac{\prod_{k=1}^{N}\left(q^{1+(N+k-2) g_{l}+g_{s}} ; q\right)_{M-1} \prod_{k=1}^{\left[\frac{N}{2}\right]}\left(q^{1+(2 k-1) g_{l}} ; q\right)_{M-1}}{\prod_{k=1}^{\left[\frac{N}{2}\right]}\left(q^{1+2(N-k) g_{l}} ; q\right)_{M-1}}$ & $2(N-1) g_{l}+2 g_{s}$ \\
\hline$C_{N}$ & $\frac{\prod_{k=1}^{N}\left(q^{1+(k-1) g_{s}+g_{l}} ; q\right)_{M-1} \prod_{k=1}^{\left[\frac{N}{2}\right]}\left(q^{1+(2 N-2 k-1) g_{s}+2 g_{l}} ; q\right)_{M-1}}{\prod_{k=1}^{\left[\frac{N}{2}\right]}\left(q^{1+2(k-1) g_{s}+2 g l} ; q\right)_{M-1}}$ & $2(N-1) g_{s}+2 g_{l}$ \\
\hline$D_{N}$ & $\left(q^{1+(N-1) g} ; q\right)_{M-1} \prod_{k=1}^{N-1}\left(q^{1+(2 k-1) g} ; q\right)_{M-1}$ & $2(N-1) g$ \\
\hline$E_{6}$ & $\left(q^{1+g}, q^{1+4 g}, q^{1+5 g}, q^{1+7 g}, q^{1+8 g}, q^{1+11 g} ; q\right)_{M-1}$ & $12 g$ \\
\hline$E_{7}$ & $\left(q^{1+g}, q^{1+5 g}, q^{1+7 g}, q^{1+9 g}, q^{1+11 g}, q^{1+13 g}, q^{1+17 g} ; q\right)_{M-1}$ & $18 g$ \\
\hline$E_{8}$ & $\left(q^{1+g}, q^{1+7 g}, q^{1+11 g}, q^{1+13 g}, q^{1+17 g}, q^{1+19 g}, q^{1+23 g}, q^{1+29 g} ; q\right)_{M-1}$ & $30 g$ \\
\hline$F_{4}$ & $\frac{\left(q^{1+g_{l}}, q^{1+g_{s}+3 g_{l}}, q^{1+2 g_{s}+3 g_{l}}, q^{1+3 g_{s}+4 g_{l}}, q^{1+3 g_{s}+5 g_{l}}, q^{1+5 g_{s}+6 g_{l}} ; q\right)_{M-1}}{\left(q^{1+2 g_{s}+6 g_{l}}, q^{1+4 g_{l}} ; q\right)_{M-1}}$ & $6 g_{s}+6 g_{l}$ \\
\hline$G_{2}$ & $\frac{\left(q^{1+g_{l}}, q^{1+g_{s}+2 g_{l}}, q^{1+2 g_{s}+3 g_{l}} ; q\right)_{M-1}}{\left(q^{1+3 g_{l}} ; q\right)_{M-1}}$ & $3 g_{s}+3 g_{l}$ \\
\hline
\end{tabular}

TABLE 2. Reduced Expressions for $\mathcal{N}_{M}$

Remark 4.5. For a root system of rank $N=1$, the terminating Aomoto-ItoMacdonald sum of Theorem 3 reduces to the identity

$$
\sum_{\substack{m \in\{0,1,2, \ldots, M\} \\ m \text { even/odd }}} q^{-g m}\left(\frac{1-q^{g+m}}{1-q^{g}}\right) \frac{\left(q^{2 g} ; q\right)_{m}}{(q ; q)_{m}}=\left(q^{1+g} ; q\right)_{M-1}
$$

where $q=\exp (\pi i /(g+M / 2))$, and the choice of summation over the even or odd nonnegative integers corresponds to the choice of $\sigma$ equal to 0 or 1 , respectively.

Remark 4.6. By summing over all minuscule weights $\sigma$ in $\mathcal{P} / \mathcal{Q}$, it is seen that in Theorem 2 we may replace the summation over the intersections $(\sigma+\mathcal{Q}) \cap \mathcal{P}^{+}$ and $(\sigma+\mathcal{Q}) \cap \mathcal{P}_{M}^{+}$in Theorems 2 and 3 respectively, by summations over the full dominant cone $\mathcal{P}^{+}$and alcove $\mathcal{P}_{M}^{+}$, upon multiplication of the corresponding constants on the r.h.s. by the index $|\mathcal{P} / \mathcal{Q}|$ (cf. Remark [2.1).

Remark 4.7. It is instructive to think of the terminating Aomoto-Ito-Macdonald sum in Theorem 3 as a trigonometric identity. Indeed, putting $q=e^{2 i \omega}$ recasts the 
summation formula in the form

$$
\begin{aligned}
\sum_{\mu \in(\sigma+\mathcal{Q}) \cap \mathcal{P}_{M}^{+}} \prod_{\alpha \in R^{+}} \frac{\sin \omega\left\langle\alpha^{\vee}, \rho_{g}+\mu\right\rangle \prod_{k=1}^{\left\langle\alpha^{\vee}, \mu\right\rangle} \sin \omega\left(\left\langle\alpha^{\vee}, \rho_{g}\right\rangle+g_{\alpha}+k-1\right)}{\sin \omega\left\langle\alpha^{\vee}, \rho_{g}\right\rangle \prod_{k=1}^{\left\langle\alpha^{\vee}, \mu\right\rangle} \sin \omega\left(\left\langle\alpha^{\vee}, \rho_{g}\right\rangle-g_{\alpha}+k\right)} \\
=2^{N(M-1)} \prod_{k=1}^{M-1} \frac{\prod_{\alpha \in R^{+}} \sin \omega\left(\left\langle\alpha^{\vee}, \rho_{g}\right\rangle+k\right)}{\prod_{\alpha \in R^{+} \backslash S} \sin \omega\left(\left\langle\alpha^{\vee}, \rho_{g}\right\rangle-g_{\alpha}+k\right)},
\end{aligned}
$$

for $\omega=\pi /\left(g_{R}+M\right)$. The positivity mentioned in Remark4.3 is immediate from the trigonometric representation. Indeed, the parameter restrictions guarantee that all arguments of the sine functions lie in the open interval $(0, \pi)$. After setting $g_{\alpha}=g$, $\forall \alpha \in R$ and performing the limit $g \downarrow 0$, the summation formula under consideration degenerates to the combinatorial identity

$$
\sum_{\mu \in(\sigma+\mathcal{Q}) \cap \mathcal{P}_{M}^{+}} \prod_{\substack{\alpha \in R^{+} \\\left\langle\alpha^{\vee}, \mu\right\rangle>0}} \frac{1+\left\langle\alpha^{\vee}, \rho\right\rangle}{\left\langle\alpha^{\vee}, \rho\right\rangle} \prod_{\substack{\alpha \in R^{+} \\\left\langle\alpha^{\vee}, \mu\right\rangle=M}} \frac{h-\left\langle\alpha^{\vee}, \rho\right\rangle}{h+1-\left\langle\alpha^{\vee}, \rho\right\rangle}=M^{N},
$$

where $\rho:=\frac{1}{2} \sum_{\alpha \in R^{+}} \alpha$ and $h:=\left\langle\alpha_{0}^{\vee}, \rho\right\rangle+1$ (the Coxeter number of $R$ ).

\section{ACKNOWLEDGMENTS}

Thanks are due to J. F. van Diejen for several helpful discussions and to the referee for careful reading of the manuscript.

\section{REFERENCES}

[AAR] G. E. Andrews, R. Askey, and R. Roy, Special Functions, Encyclopedia of Mathematics and its Applications 71, Cambridge University Press, Cambridge, 1999. MR 2000g:33001

[A] K. Aomoto, On elliptic product formulas for Jackson integrals associated with reduced root systems, J. Algebraic Combin. 8 (1998), 115-126. MR 2000b:33012

[B] N. Bourbaki, Groupes et algèbres de Lie, Chapitres 4-6, Hermann, Paris, 1968. MR 39:1590

[D] J. F. van Diejen, On certain multiple Bailey, Rogers, and Dougall type summation formulas, Publ. Res. Inst. Math. Sci. 33 (1997), 483-508. MR 98j:33011

[DS] J. F. van Diejen and V. P. Spiridonov, An elliptic Macdonald-Morris conjecture and multiple modular hypergeometric sums, Math. Res. Lett. 7 (2000), 729-746. MR 2002m:33022

[DV] J. F. van Diejen and L. Vinet, The quantum dynamics of the compactified trigonometric Ruijsenaars-Schneider model, Commun. Math. Phys. 197 (1998), 33-74. MR 2000k:81123

[GR] G. Gasper and M. Rahman, Basic Hypergeometric Series, Encyclopedia of Mathematics and its Applications 35, Cambridge Univ. Press, Cambridge, 1990. MR 91d:33034

[G1] R. A. Gustafson, Multilateral summation theorems for ordinary and basic hypergeometric series in $\mathrm{U}(n)$, SIAM J. Math. Anal. 18 (1987), 1576-1596. MR 90e:33015

[G2] - The Macdonald identities for affine root systems of classical type and hypergeometric series very-well-poised on semisimple Lie algebras, Ramanujan International Symposium on Analysis (Pune, 1987), 185-224, Macmillan of India, New Delhi, 1989. MR 92k:33015

[I1] M. Ito, A theta product formula for Jackson integrals associated with root systems, Proc. Japan Acad. Ser. A Math. Sci. 73 (1997), 60-61. MR 99b:33043

[I2] - On a theta product formula for Jackson integrals associated with root systems of rank two, J. Math. Anal. Appl. 216 (1997), 122-163. MR 99e:33023

[I3] Symmetry classification for Jackson integrals associated with irreducible root systems, Compositio Math. 129 (2001), 325-340. MR 2002m:33021] 
[K] J. Kaneko, q-Selberg integrals and Macdonald polynomials, Ann. Sci. École Norm. Sup. (4) 29 (1996), 583-637. MR 98k:33026

[M1] I. G. Macdonald, Affine root systems and Dedekind's $\eta$-function, Invent. Math. 15 (1972), 91-143. MR 50:9996

[M2] The Poincaré series of a Coxeter group, Math. Ann. 199 (1972), 161-174. MR 48:433

[M3] - Some conjectures for root systems, SIAM J. Math. Anal. 13 (1982), 988-1007. MR 84h:17006a

[M4] A formal identity for affine root systems, preprint, 1996

$[\mathrm{M}]$ S. C. Milne, Basic hypergeometric series very well-poised in $\mathrm{U}(n)$, J. Math. Anal. Appl. 122 (1987), 223-256. MR 88h:33007

[ML] S. C. Milne and G. M. Lilly, Consequences of the $A_{l}$ and $C_{l}$ Bailey transform and Bailey lemma, Formal Power Series and Algebraic Combinatorics (Montreal, PQ, 1992). Discrete Math. 139 (1995), 319-346. MR 96g:33025

[O] E. M. Opdam, Some applications of hypergeometric shift operators, Invent. Math. 98 (1989), 1-18. MR 91h:33024

[R] H. Rosengren, Karlsson-Minton type hypergeometric functions on the root system $C_{n}$, J. Math. Anal. Appl. 281 (2003), 332-345.

[S] M. Schlosser, Multidimensional matrix inversions and multiple basic hypergeometric series associated to root systems, Special Functions and Differential Equations (Madras, 1997), 25-30, Allied Publ., New Delhi, 1998. MR 99i:33024

Instituto de Matemática y Física, Universidad de Talca, Casilla 747, Talca, Chile

E-mail address: anace@inst-mat.utalca.cl 